\title{
GEANT4 SIMULATION AND THEORETICAL STUDIES OF A HELICAL COOLING CHANNEL
}

\begin{abstract}
We present a conceptual design and detailed GEANT4 simulation of a helical ionization cooling channel proposed by Ya. Derbenev, which is able to cool in 3 dimensions (3D). The lattice of the channel consists of a long solenoid $(\mathrm{B}=5 \mathrm{~T}, \mathrm{~L}=72 \mathrm{~m}, \mathrm{R}=70 \mathrm{~cm}$ ) and a transverse dipole magnetic field which rotates with a period of $1.8 \mathrm{~m}$ and an amplitude of $0.3 \mathrm{~T}$. The cooling is achieved using lithium hydride wedge absorbers. Re-acceleration is performed by 201.25 MHz RF cavities which provide a $6.2 \mathrm{MeV}$ per cooling cell. The parameters of the muon beam at the end of the channel are: transmission $85 \%$, transverse and longitudinal emittances $0.6 \mathrm{~cm}$ and $2 \mathrm{~cm}$, respectively.
\end{abstract}

V. Balbekov, V.D. Elvira, P. Lebrun, P. Spentzouris, FNAL, Batavia, IL 60510, USA

\section{INTRODUCTION}

Over the last few years, the Neutrino Source/Muon Collider $(\nu / \mu)$ Collaboration has developed options for muon cooling based on alternating-solenoids or long solenoids [1, $2]$. These channels cool in two dimensions (2-D) transversely by a factor of $\approx 5-7$ with a total transmission of 50-75\%. Recently, Y. Derbenev [3] suggested the possibility of achieving 3-D cooling (transverse and longitudinal) for muons on a helical orbit. Towards that goal, he developed a linear theory of ionization cooling along a helical orbit in a dipole helical field, combined with a solenoidal field. Particles with different $p_{z}$ would have different location and, therefore, would go through a different amount of wedge shaped absorber material. This cooling mechanism is known as "emittance exchange".

In this paper, we present a brief summary of a GEANT4 simulation $[4,5]$ of a cooling channel based on the helical cooling concept. (GEANT4 performs tracking of muons through electromagnetic fields and matter.) We also discuss the theoretical studies and the simple simulations reported in Ref. [6], where this channel was originally proposed.

\section{DESCRIPTION OF THE HELICAL COOLING CHANNEL}

The helical cooling channel introduced in Ref. [6] is $72 \mathrm{~m}$ long, and consists of 40 unit cells which repeat periodically along the direction of the beam $(\hat{z})$. A unit cell, $1.8 \mathrm{~m}$ long, consists of (see Figure 1): two wedge absorbers a few $\mathrm{cm}$ thick, two RF short linacs in between the absorbers, a solenoid which surrounds the absorbers and RF cavities, a dipole field of constant magnitude which rotates about the z-axis with a periodicity equal to the unit cell length.

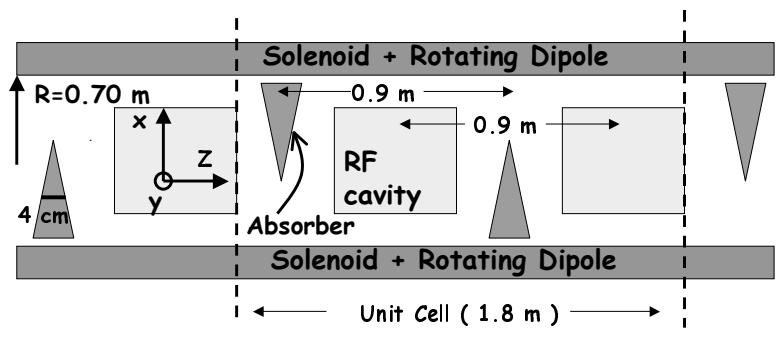

Figure 1: A unit cell is shown in between dashed lines, containing two wedge absorbers and two RF short linacs.

\subsection{The Magnetic Field}

In the laboratory frame, a helical magnetic field is expressed as: $B_{x}=B_{T}\left[\sin (2 \pi z / L)+O\left(r^{2} / L^{2}\right)\right]$, $B_{y}=B_{T}\left[\cos (2 \pi z / L)+O\left(r^{2} / L^{2}\right)\right], B_{z}=B_{0}[1+$ $\left.O\left(B_{T} r / B_{0} L\right)\right]$, where $B_{0}$ is the field on axis of an infinite solenoid, $B_{T}$ is the amplitude of the transverse dipole field, and $r$ is the particle radius.

A helical field may be generated by tilting the coils of a solenoid with a given periodicity [6]. For the GEANT4 simulation presented here, the solenoidal field on axis (generated from current distributions) is $B_{0}=5 \mathrm{~T}$ and the dipole field is $0.3 \mathrm{~T}$ in amplitude with a $L=1.8 \mathrm{~m}$ period. The dipole was not created from currents but added analytically. In Ref. [6], the equations of motion of a particle in a helical field were solved under different approximations $\left(B_{z}=B_{0}\right.$ in all of them): (a) paraxial (higher order terms in $\vec{B}$ are neglected), (b) non-linear excluding dependence of longitudinal velocity on transverse momentum $\left(v_{z}=v\right)$, (c) nonlinear including $v_{z}=f\left(p_{T}\right)$, (d) same as (c) plus an $E$ $p_{T}$ correlation term, (e) same as (c) but without a dipole field and no wedges (no emittance exchange). While the change in transmission and emittance is negligible when going from (a) to (b), there is a significant change when going to (c) and then to (d). Although our GEANT4 simulation includes a $B_{z}=B(z, r)$ and neglects higher orders in $B_{x}$, and $B_{y}$, the results can be directly compared to those from case (d) in Ref. [6].

To avoid the excitation of betatron oscillations in the beginning and the end of the channel, the dipole field was turned on/off adiabatically. The maximum amplitude of $0.3 \mathrm{~T}$ is reached after 8 periods $(14.4 \mathrm{~m})$, kept constant for 24 periods, and decreased to zero over the last 8 periods.

\subsection{The Absorber}

A particle initially at the origin of the channel with zero transverse momentum and $E=E_{\text {ref }}$ (reference energy: energy necessary for a particle to travel with $V_{z}$ equal to propagation velocity of the RF wave), describes a quasi- 
periodic orbit with a modulation on the particle radius which depends on $E_{\text {ref }}$ (see Figure 2). The closer to resonance $\left(E_{\text {res }}=442 \mathrm{MeV}\right)$, the larger the modulation. The reference energy has to be small enough to reduce the modulations to acceptable levels, and large enough to maintain a reasonable bucket area. The optimal value consistent with the neutrino source design is $E_{\text {ref }}=260 \mathrm{GeV}$.

The inset plot in Figure 2 shows the best positions to place the wedge absorbers, separated by $0.9 \mathrm{~m}$. These locations correspond to the points of maximum dipole field. The wedges, made of lithium hydride $(\mathrm{LiH})$, are located as specified in Figure 3. The thickness seen by the reference particle is $4 \mathrm{~cm}$, at a distance of $3.9 \mathrm{~cm}$ from the system $z$-axis. The distance between the position of the reference particle and the wedge inner edge is $15 \mathrm{~cm}$, which corresponds to $3 \sigma_{x}$ ( $\sigma_{x}$ is the size of the beam). At $260 \mathrm{MeV}$ with a $d E / d x \approx 1.55 \mathrm{MeV} / \mathrm{cm}$, the reference particle losses about 6.2 MeV per wedge.

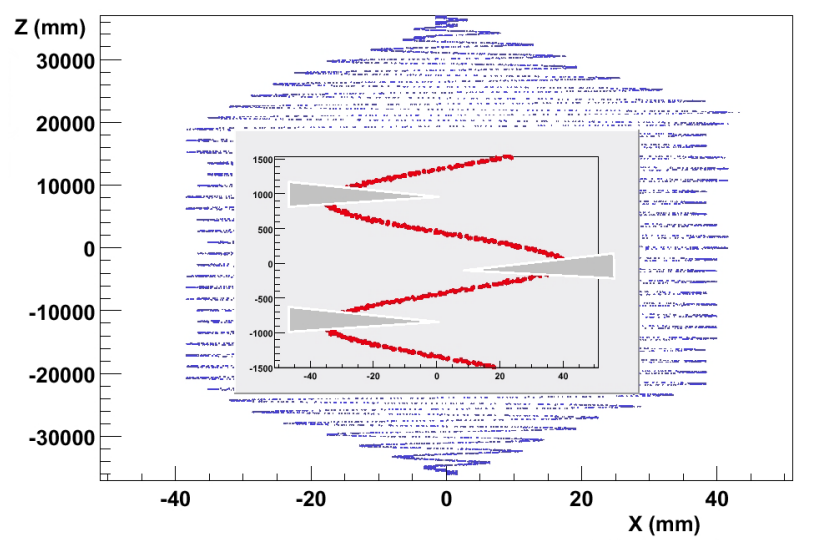

Figure 2: Trajectory of the reference particle ( $x$ versus $z$ ). The inset plot shows the location of the absorbers in a larger scale.

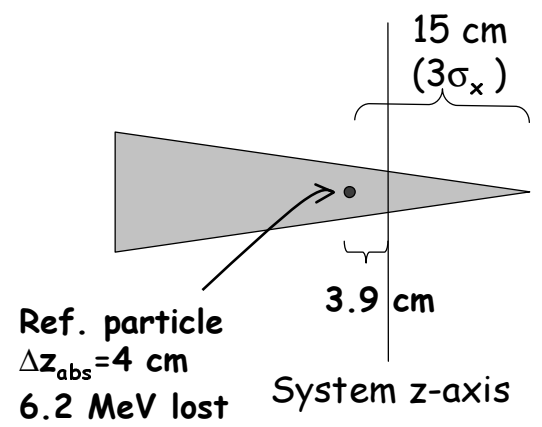

Figure 3: Lithium hydride $(\mathrm{LiH})$ wedges size and location

\subsection{The rf System}

For simplicity, we modeled the short linacs with just one $\nu=201 \mathrm{MHz}$ thin cavity ( $1 \mathrm{~cm}$ long), which, at a synchronous phase of $30^{\circ}$, provides $6.2 \mathrm{MeV}$ per cooling cell. The linac is placed immediately after the absorber. The RF system was tuned by adjusting the phases of each cavity (around $30^{\circ}$ ) so that $p_{z}$ and $p_{T}$ as a function of $z$ are the same to within $1 \mathrm{MeV}$ before and after the absorbers are placed. Random physics processes like multiple scattering and straggling were turned off to tune the phases.

\section{THE BEAM}

Initially, the position and momentum distributions of the Larmor centers are Gaussian around zero with $\sigma_{x L i}=$ $\sigma_{y L i}=3.5 \mathrm{~cm}, \sigma_{p x L_{i}}=\sigma_{p y L_{i}}=48.7 \mathrm{MeV}$, as well as the initial time distribution with $\sigma_{c t_{i}}=10 \mathrm{~cm}$. The initial energy is also Gaussian distributed around the $260 \mathrm{MeV}$ reference energy with $\sigma_{E_{i}}=25 \mathrm{MeV}$. The Gaussian beam was corrected for angular momentum $\left(x-p_{y}\right.$ and $y-p_{x}$ correlations), and injected inside the solenoid at the origin of the channel ( $z=-36 \mathrm{~m})$.

\section{RESULTS}

The evolution of the 3-D, transverse, longitudinal emittance, and transmission (ratio between the number of particles in the beginning and the end of the channel) was studied as a function of the channel length for different simulation conditions: (i) the beam as described in Sec. 3, (ii) the same beam with the addition of the $E-p_{T}$ correlation $\langle E\rangle=E_{\text {ref }} \sqrt{1+\left(p_{T} / m_{\mu} c\right)^{2}}$, which may be generated at the buncher level. (The buncher is the accelerator section which precedes the cooling channel in the neutrino factory.)

In (i) the losses are large in the beginning of the channel because the longitudinal emittance increases as the dipole field turns on and the beam acquires transverse momentum. At the end, due to the emittance exchange mechanism, the longitudinal emittance is reduced back to approximately the initial value.

Figure 4 shows the $E-\langle E\rangle$ versus $c t-\langle c t\rangle$ distribution at the beginning (bottom left) and at the end (bottom right) of the channel. Due to the dependence of longitudinal velocity on transverse momentum, there are two regions of stability. Since $p_{T}$ grows fast near resonance, $p_{z}$ increases when $E$ increases, and $p_{z}$ is the same at $E \approx 260 \mathrm{GeV}$ and $E \approx 400 \mathrm{GeV}$. Figure 4 also illustrates on transverse cooling. The top left plot is the initial $p_{x}$ versus $x$ distribution and the top right plot is the same distribution at the end of the channel. While $\sigma_{p x}$ decreases fast as a function of $z, x$ and $y$ stay the same. The channel reduces $\sigma_{p_{x}}, \sigma_{p_{y}}$, and the Larmor radii, but not the Larmor centers. Figure 5 shows emittances and transmission versus $z$ for case (ii). The introduction of the $E-p_{T}$ correlation helps to maintain longitudinal stability. Losses are smaller than in (i), the longitudinal emittance decreases monotonically, and the final transverse and longitudinal emittances are smaller. Cuts: $-400 \mathrm{~mm}<|c t-\langle c t\rangle|<600 \mathrm{~mm}$ and $\left|E-E_{\text {ref }}\right|<75 \mathrm{MeV}$ are applied to remove the secondary stable region for the emittance and transmission calculation. Since the cuts were applied over the whole channel length, the transmission and emittance values displayed in Figure 5 do not represent the real initial parameter values of the beam. 
Table 1 contains results on initial and final emittances, and transmission for simulations (i), (ii) (see Ref. [7]), and (d) (see Ref. [6]). The agreement between the GEANT4 simulation (ii) and the simulation (d) in Ref. [6] is excellent.

Table 2 shows results from the simulation in Ref. [6]for cases (a) through (e). The inclusion of all non-linearities reduces the transmission significantly, from $92.2 \%$ to $60.5 \%$. A large fraction of the particles can be recovered by introducing a $E-p_{T}$ correlation as in (d). Without emittance exchange (e), the longitudinal emittance grows to $3.31 \mathrm{~cm}$ at the end of the channel, although the transverse parameters and the transmission are better than in (d).
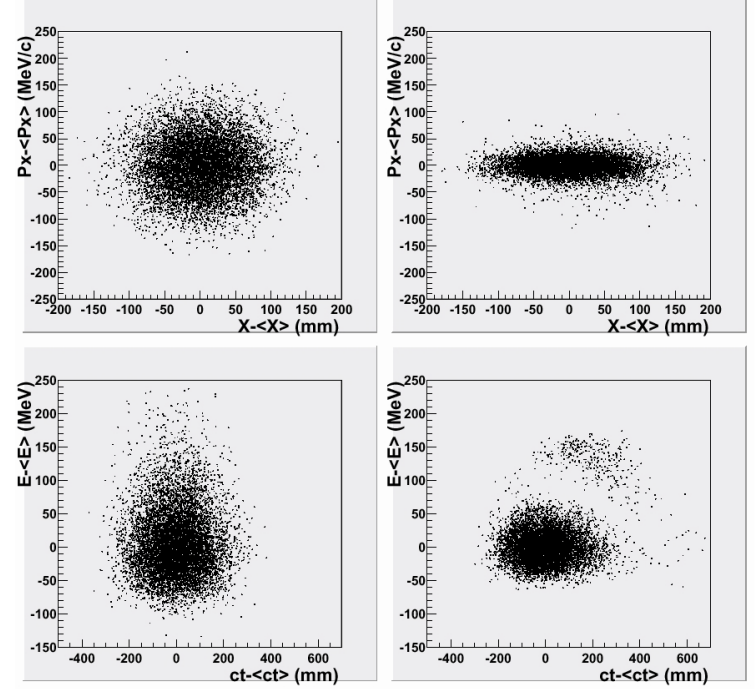

Figure 4: Top (bottom): $p_{x}$ versus $x$ (E- $\langle E\rangle$ versus $c t-$ $\langle c t\rangle)$ at the beginning, left, and the end, right, of the cooling channel.

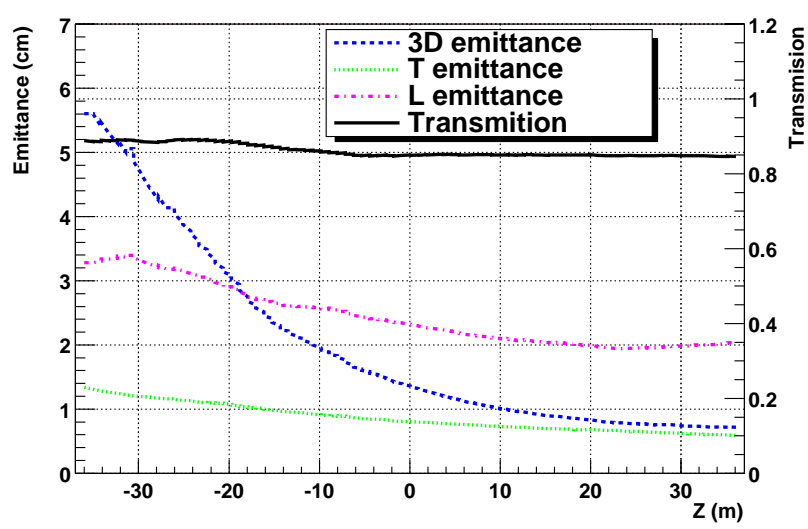

Figure 5: Emittance and transmission versus $z$. Multiple scattering is turned on and there is an initial correlation between $E$ and $p_{T}$ case (ii).

\section{CONCLUSIONS}

A helical cooling channel has been studied and simulated. A version of this helical channel could eventually
Table 1: Initial and final emittance and transmission for simulations (i), (ii) and (d).

\begin{tabular}{|l|l|l|l|l|}
\hline & $\begin{array}{l}\text { Trans } \\
\%\end{array}$ & $\begin{array}{l}\varepsilon_{3 D} \\
\mathbf{c m}^{3}\end{array}$ & $\begin{array}{l}\varepsilon_{T} \\
\mathbf{c m}\end{array}$ & $\begin{array}{l}\varepsilon_{L} \\
\mathbf{c m}\end{array}$ \\
\hline Initial (i)/(d) & & 5.37 & 1.5 & 2.37 \\
\hline Final (i) & 65 & 0.83 & 0.56 & 2.57 \\
\hline Initial (ii) & & 10.52 & 1.5 & 4.63 \\
\hline Final (ii) & 85.0 & 0.72 & 0.59 & 2.04 \\
\hline Final (d) & 83.0 & 0.75 & 0.605 & 2.04 \\
\hline
\end{tabular}

Table 2: Results from the simple simulation in Ref. [6].

\begin{tabular}{|l|l|l|l|l|}
\hline & $\begin{array}{l}\text { Trans } \\
\%\end{array}$ & $\begin{array}{l}\varepsilon_{3 D} \\
\mathbf{c m}^{3}\end{array}$ & $\begin{array}{l}\varepsilon_{T} \\
\mathbf{c m}\end{array}$ & $\begin{array}{l}\varepsilon_{L} \\
\mathbf{c m}\end{array}$ \\
\hline Initial (i)/(d) & & 5.37 & 1.5 & 2.37 \\
\hline Final (a) & 93.4 & 0.417 & 0.458 & 1.99 \\
\hline Final (b) & 92.2 & 0.489 & 0.501 & 1.95 \\
\hline Final (c) & 60.5 & 0.650 & 0.513 & 2.47 \\
\hline Final (d) & 83.0 & 0.747 & 0.605 & 2.04 \\
\hline Final (e) & 92.9 & 0.540 & 0.404 & 3.31 \\
\hline
\end{tabular}

be used to replace the first section of a channel based on the double flip concept [7] to keep the longitudinal emittance under control and increase transmission. Although this is an interesting option, the technical challenges associated with producing a strong enough rotating dipole field, among other issues, are significant.

\section{REFERENCES}

[1] The Muon Collider/Neutrino Source Collaboration, "A Feasibility Study of a Neutrino Source based on a Muon Storage Ring" (based on a Fermilab site). Submitted to Phys. Rev. ST - Accelerators and Beams. FERMILAB-PUB00-108-E (2000).

[2] The document on a feasibility study of a neutrino source in Brookhaven National Laboratory is currently in preparation.

[3] "Conceptual Studies on Ionization Cooling of Muon Beam", Ya. Derbenev. MuCool note \#185, 11/27/00.

[4] http://wwwinfo.cern.ch/asd/geant4/geant4.html

[5] "Simulation of a Helical Channel Using GEANT4", V. D Elvira, P. Lebrun, P. Spentzouris. MuCool note \#193, $8 / 6 / 00$.

[6] "Helical channel studies and simulation", V. Balbekov. MuCool note \#146, 8/6/00.

[7] "The Double Flip Cooling Channel", V. Balbekov, V. D. Elvira, et al. MuCool note \#203, 8/6/00.

MuCool notes are available at http://www-mucool.fnal.gov/notes/notes.html 